\title{
Genetic study of indirect inguinal hernia
}

\author{
Yaoqin Gong, Changshun Shao, Qian Sun, Binxi Chen, Yuan Jiang, Chenhong Guo, \\ Jianjun Wei, Yishou Guo
}

\begin{abstract}
We performed a genetic analysis of 280 families with congenital indirect inguinal hernia ascertained in Shandong province. The multifactorial threshold model and segregation analysis were applied to these families to investigate the mode of inheritance of congenital indirect inguinal hernia. Our results indicate that congenital indirect inguinal hernia is not compatible with a multifactorial threshold model, and the frequent vertical transmission and high segregation ratio suggest autosomal dominant inheritance with incomplete penetrance and sex influence. Through further pedigree analysis of the multiple case families with at least two closely related affected members, we noted preferential paternal transmission of the disease gene, which might suggest the role of genomic imprinting in the aetiology of this condition.
\end{abstract}

(f Med Genet 1994;31:187-192)

Inguinal hernia (IH) is a common developmental anomaly; its incidence has been estimated to be 6 to $12.5 \%$ in the different general populations studied. ${ }^{1}$ IH can be classified into direct inguinal hernia (DIH) and indirect inguinal hernia (IIH), the latter being far more common than the former. IIH is further subdivided into congenital and acquired IIH. Congenital IIH is generally considered to result from a congenital weakness in the internal ring and a persistent processus vaginalis and can be detected during the first months or years after birth.

Although there has been considerable evidence suggesting the role of genetic factors in the aetiology of $\mathrm{IH},{ }^{23}$ its mode of inheritance remains controversial. Hypotheses proposed include (1) autosomal dominant inheritance with incomplete penetrance, ${ }^{34}$ (2) autosomal dominant inheritance with sex influence, ${ }^{5}$ (3) $\mathrm{X}$ linked dominant inheritance, ${ }^{6}$ and (4) polygenic inheritance. ${ }^{7-9}$

In a recent survey of major genetic disease in the general population of Shandong province, ${ }^{10}$ we identified 280 families with congenital IIH. We applied the multifactorial threshold (MFT) model and segregation analysis to these data to investigate the mode of inheritance in congenital IIH. Our results indicate that congenital IIH is not compatible with an MFT model, and the frequent vertical transmission and high segregation ratio suggest autosomal dominant inheritance with incomplete penetrance and sex influence in familial IIH. Through further pedigree ana- lysis of multiple case families with at least two closely related affected members, we noted the preferential paternal transmission of the disease gene, suggesting the role of genomic imprinting in the aetiology of this condition.

\section{Materials and methods \\ FAMILIES}

The cases used in this study were identified in the project "Survey of Major Genetic Diseases in Shandong Province" which was carried out in the five year period from 1985 to $1990 .{ }^{10}$ The survey sites were located in 11 prefectures and covered a population of about one million. The work for IIH was carried out in a two stage procedure. During the first stage the patients with IIH were registered by the trained village or community doctors. Each patient identified in the first step was regarded as a proband (index patient). In the second stage, a thorough clinical examination was conducted on these index patients and their affected first degree relatives, then the pedigree was drawn. Among 392 index patients, 52 index patients with acquired IIH were excluded from further analysis. The 340 probands (319 male, 21 female) had all been operated on by 5 years. These probands were from 280 families. The hernia occurred on the right side in 138 probands, on the left side in 84 probands, and on both sides in the other 118 probands.

\section{GENETIC ANALYSIS}

The observed characteristics of familial aggregation of IIH were compared with the expected values based on an MFT model to discriminate between multifactorial and mendelian inheritance. ${ }^{11-13}$ These characteristics include correlations of the proband's sex to the recurrence risk of relatives, and the severity of the proband's defect to the recurrence risk of relatives. These correlations were evaluated in the first degree relatives of different types of probands.

Classical segregation analysis was performed on 341 nuclear families. According to the parents' mating type, we divided nuclear families into three groups: (1) one parent affected ( $\mathrm{U} \times \mathrm{A}$ matings), (2) both parents normal with positive family history, that is, one parent has a first or second degree relative with $\mathrm{IIH}(\mathrm{U} \times \mathrm{U}(\mathrm{f})$ matings), and (3) both parents normal ( $U \times U$ matings). We used the following distributions for the expectation of families of size s with only one affected sib (1) and with more than one affected sib (2). 


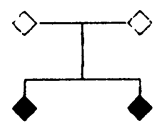

Family A

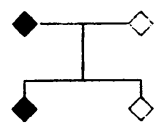

Family B

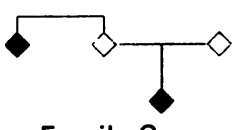

Family C

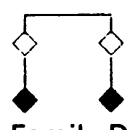

Family D

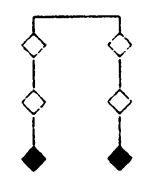

Family E
Figure 1 Families with multiple cases of IIH. this method, the fit of a parametric value, say $\mathrm{p}$, to the data is tested by $\chi \mathrm{p}^{2}=(\mathrm{Up})^{2} / \mathrm{Kpp}$, with $1 \mathrm{df}$, where $\mathrm{Up}$ is the ML score and $\mathrm{Kpp}$ its variance. The iteration procedure will lead to the best estimate of the parameter. ${ }^{14}$

\section{ANALYSIS OF PARENTAL ORIGIN OF THE DISEASE} GENE

The criteria for the multiple case families used
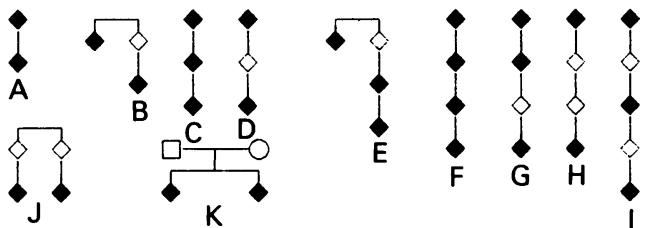

Figure 2 Illustration of transmission types. Families $A$ and $B$, vertical transmission through two generations. Families $C, D$, and $E$, vertical transmission through three generations. Families $F, G$, and $H$, vertical transmission through four generations. Family I, vertical transmission through five generations. Families $\mathcal{f}$ and $K$, non-vertical transmission.

$$
\begin{aligned}
& \mathrm{P}(\mathrm{r}=1)=\left\{\operatorname{sp} \pi\left[\mathrm{x}+(1-\mathrm{x}) \mathrm{q}^{\mathrm{s}-1}\right]\right\} / \\
& \left\{\mathrm{sp} \pi \mathrm{x}+(1-\mathrm{x})\left[1-(1-\mathrm{p} \pi)^{s}\right]\right\} \\
& \mathrm{P}(\mathrm{r}>1)=\left\{(1-\mathrm{x})\left[1-(1-\mathrm{p} \pi)^{\mathrm{s}}-\mathrm{sp} \pi \mathrm{q}^{\mathrm{s}-1}\right]\right\} / \\
& \left\{\mathrm{sp} \pi \mathrm{x}+(1-\mathrm{x})\left[1-(1-\mathrm{p} \pi)^{\mathrm{s}}\right]\right\}
\end{aligned}
$$

where $s$ is the sibship size, $r$ the number of affected sibs, $p$ the segregation frequency, $q$ equal to $1-p, x$ the frequency of sporadic cases, and $\pi$ the probability of ascertainment. The values of $\mathrm{p}$ and $\mathrm{x}$ were estimated under incomplete selection (with $\pi=0 \cdot 78$ ) by the maximum likelihood (ML) method. ${ }^{14}$ With

Table 1 Percentages of different types of families among the multiple case families

\begin{tabular}{llll}
\hline Transmission & No of generations & Family type & No of families (\%) \\
\hline Vertical & 2 & A & 23 \\
& & B & 15 \\
& 3 & C & $38(48 \cdot 7)$ \\
& & D & 3 \\
& 4 & E & 2 \\
& & F & $12(15 \cdot 4)$ \\
& & G & 1 \\
Non-vertical & 5 & H & 2 \\
& & I & $4(5.1)$ \\
& & K & 15 \\
& & & 8 \\
& & & $23(29 \cdot 5)$ \\
\hline
\end{tabular}

Table 2 Risk to first degree relatives by proband's sex*

\begin{tabular}{llll}
\hline Proband's sex & Total 1st degree relatives & Affected 1st degree relatives & Incidence (\%) \\
\hline Male & 1030 & 64 & 6.21 \\
Female & 72 & 5 & 6.94 \\
\hline
\end{tabular}

${ }^{*} \mathrm{p}>0.05$.

Table 3 Risk to first degree relatives by IIH type of probands*

\begin{tabular}{llll}
\hline IIH type of proband & Total 1st degree relatives & Affected 1st degree relatives & Incidence \\
\hline Bilateral & 253 & 16 & 0.0632 \\
Unilateral & 509 & 32 & 0.0629 \\
$\quad$ Right & 340 & 21 & 0.0618 \\
Left & 849 & 53 & 0.0624 \\
Total & & &
\end{tabular}
to analyse the parental origin of the IIH gene were: (1) there were at least two affected members; (2) affected members were distributed in at least two sibships; and (3) the relationship between them was up to third degree. The illustration of how these criteria were used are shown in fig 1 . Using these criteria, families B, $C$, and $D$ in fig 1 have been included in our study, while family $\mathrm{A}$ and $\mathrm{E}$ have been excluded.

According to the above criteria, a total of 128 families was identified, of which 70 families were selected from the 280 families, the other $\mathbf{5 8}$ families coming from previously published studies, ${ }^{15}$ which were ascertained through 58 probands who underwent operations in Jinan Children's Hospital during 1989 to 1991. Phenotypically normal carriers who had carried and transmitted the disease gene to the next generation were identified by pedigree analysis. The sex of each carrier and affected parent was recorded.

\section{Results}

PEDIGREE ANALYSES

Of the 280 families, there was a positive history in 78 families $(27.85 \%)$. In 202 families $(72 \cdot 14 \%)$, the index patient was the only known affected family member. The illustration of different family types is shown in fig 2 . In 55 of the 78 families with a positive history, the trait was transmitted vertically (table 1). Direct male to male transmission was noted in 29 families. The vertical and male to male transmission of the trait in these families suggests that familial IIH is inherited in autosomal dominant fashion.

TEST OF THE MFT MODEL

In this data set, the sex ratios in probands and affected subjects were $15 \cdot 32(319: 21)$ and 13.36 (374:28), respectively. According to the MFT model, when the incidence is higher in one sex than in the other, the less affected sex would be expected to show a higher recurrence risk in their first degree relatives compared to the more affected sex. However, as is shown in table 2, the incidence of IIH in the first degree relatives of female probands was not significantly higher than that of male probands $(\mathrm{p}>0.05)$.

Another prediction with a similar theoretical basis is that the more severely affected probands would be expected to show a higher risk in their first degree relatives compared to the less severely affected probands. In this study, the incidence of IIH in the first degree 
Table 4 Distributions of affected males ( $r$ ) among male sibs ( $s$ )

\begin{tabular}{|c|c|c|c|c|c|c|c|c|c|c|c|c|}
\hline \multicolumn{4}{|l|}{$U X U$} & \multicolumn{5}{|c|}{$U X U(f)$} & \multicolumn{4}{|c|}{$U X A$} \\
\hline$s / r$ & 1 & 2 & Total & $s / r$ & 1 & 2 & 3 & Total & $s / r$ & 1 & 2 & Total \\
\hline $\begin{array}{l}1 \\
2 \\
3 \\
4 \\
5 \\
\text { Total }\end{array}$ & $\begin{array}{r}114 \\
49 \\
27 \\
10 \\
2 \\
202\end{array}$ & $\begin{array}{l}5 \\
2 \\
1\end{array}$ & $\begin{array}{r}114 \\
54 \\
29 \\
11 \\
2 \\
210\end{array}$ & $\begin{array}{l}1 \\
2 \\
3 \\
4 \\
5\end{array}$ & $\begin{array}{r}44 \\
20 \\
12 \\
5 \\
2 \\
83\end{array}$ & $\begin{array}{l}9 \\
2\end{array}$ & $\begin{array}{l}1 \\
1 \\
2\end{array}$ & $\begin{array}{r}44 \\
29 \\
14 \\
6 \\
3 \\
96\end{array}$ & $\begin{array}{l}1 \\
2 \\
3 \\
4 \\
5\end{array}$ & $\begin{array}{r}21 \\
5 \\
4 \\
1 \\
1 \\
32\end{array}$ & $\begin{array}{l}1 \\
1 \\
4\end{array}$ & $\begin{array}{r}21 \\
7 \\
4 \\
2 \\
2 \\
36\end{array}$ \\
\hline
\end{tabular}

relatives of bilateral probands was not significantly higher than that of unilateral probands (table 3). A similar result was observed in the comparison of probands with right IIH and left IIH.

\section{SEGREGATION ANALYSIS}

The above analyses suggest that dominant inheritance may be more likely in these families. As noted in table 4, we have 36 nuclear families with one parent affected and 306 families with both parents unaffected, of which 96 had a positive family history; thus complete penetrance can be ruled out for the dominant transmission model. As most cases of IIH are males, segregation analyses were performed on male sibships only. The results of segregation analyses for the three groups are summarised in table 5 .

Since the proportion of sporadic cases, if any, may be unique for the families with one parent affected, $\mathrm{p}$ and $\mathrm{x}$ were simultaneously estimated from the data. The iteration procedure resulted in the final estimates of $p=0.225$ and $x=0.013$, showing that among non-sporadic cases the segregation frequency is 0.225 . If these families were considered as representing dominant inheritance, the penetrance is 0.225 / $0 \cdot 5=0 \cdot 45$.

For the sibships of UXU (f), $p=0.225$ and $x=0.013$ have an excellent fit to the data, with $\chi \mathrm{p}^{2}=0.032$ and $\chi \mathrm{x}^{2}=0.041$. This is consistent with the speculation that familial IIH shows dominant inheritance with incomplete penetrace.

For those families with a negative family history, the proportion of sporadic cases is far from zero $\left(\chi x^{2}=13.28, p<0.01\right)$. The best estimate of $\mathrm{x}$ is $\mathbf{0 . 5 9 9}$ for this group of families.

PARENTAL ORIGIN OF THE IIH GENE

A total of 159 sibships with affected or carrier parents was included in the 128 pedigrees selected. In 58 of 60 sibships the father was the affected parent, while the mother was affected in only two sibships. In 66 of 99 sibships the phenotypically normal father carried and transmitted the disease gene; in the other 33 sibships the disease genes were transmitted

Table 5 Segregation analyses of IIH families

\begin{tabular}{llllll}
\hline Group & No of families & $p$ & $x$ & $\chi p^{2}$ & $\chi x^{2}$ \\
\hline $\mathrm{U} \times \mathrm{U}$ & 210 & $0 \cdot 225$ & 0 & $12 \cdot 26$ & $13 \cdot 28$ \\
$\mathrm{U} \times \mathrm{U}(\mathrm{f})$ & 210 & $0 \cdot 225$ & 0.599 & $0 \cdot 04$ & 0 \\
$\mathrm{U} \times \mathrm{A}$ & 96 & $0 \cdot 5$ & 0 & $23 \cdot 12$ & $31 \cdot 02$ \\
& 96 & $0 \cdot 225$ & $0 \cdot 013$ & $0 \cdot 03$ & $0 \cdot 04$ \\
& 36 & 0.5 & 0 & $7 \cdot 54$ & $7 \cdot 27$ \\
\hline
\end{tabular}

Table 6 Number of sibships with affected or carrier parent

\begin{tabular}{llll}
\hline Parent & Affected & Carrier & Total \\
\hline Father & 58 & 66 & 124 \\
Mother & 2 & 33 & 35 \\
Total & 60 & 99 & 159
\end{tabular}

Table 7 Number of sibships with affected and carrier grandparents

\begin{tabular}{llll}
\hline Grandparent & Affected & Carrier & Total \\
\hline Grandfather & 32 & 3 & 35 \\
Grandmother & 2 & 1 & 3 \\
Total & 34 & 4 & 38
\end{tabular}

Table 8 The parental origin of the IIH gene by pedigree analysis

\begin{tabular}{lll}
\hline Group & Male affected & Female affected \\
\hline Total & 177 & 11 \\
Paternal & $140(79 \cdot 1 \%)$ & $6(54 \cdot 5 \%)$ \\
Affected father & 68 & 3 \\
Carrier father & 72 & 3 \\
Maternal & $37(20 \cdot 9 \%)$ & $5(45 \cdot 5 \%)$ \\
Affected mother & 1 & 0 \\
Carrier mother & 36 & 5 \\
\hline
\end{tabular}

from the carrier mother. A striking difference exists in the sex distribution of the affected parents and carriers (table 6).

Data on the grandparental generation are shown in table 7 . Though not complete, they show a similar tendency of distorted sex ratio to that of the parental generation.

The results described above suggest that the affected persons might have inherited the IIH gene more frequently from their father than from their mother. Therefore, the parental origin of the IIH gene was determined separately for affected males and females. The results show that the majority of the affected males $(79 \cdot 1 \%$, table 8$)$ inherited the IIH gene from their father $(p<0.01)$. However, the fact that females rarely develop IIH means that there are more families with an affected father than with an affected mother and makes it invalid to compare the number of affected fathers with the number of affected mothers to determine the origin of the IIH gene. We therefore compared only the numbers of carrier fathers and carrier mothers. Of the 108 affected males with a carrier parent, 72 had a carrier father and 36 had a carrier mother, the former being far more than the latter $\left(\chi^{2}=12.00, \mathrm{p}<\cdot 05\right)$. Unfortunately, the female affected sample is too small to be analysed.

\section{Discussion}

Most cases of IIH arise from retention or imperfect obliteration of the processus vaginalis, the embryological outpocketing of peritoneum that precedes testicular descent into the scrotum. The testes originate along the urogenital ridge in the retroperitoneum and $\mathrm{mi}-$ grate caudally during the second trimester of pregnancy to arrive at the internal inguinal 
(abdominal) ring at about the sixth month of intrauterine life. During the last trimester, they proceed through the abdominal wall via the inguinal canal and descend into the scrotum, the right slightly later than the left. The processus vaginalis then normally disappears postnatally except for that portion surrounding and serving as a covering for the testis. Failure of this obliterative process results in congenital IIH. Acquired IIH probably results from the stress of muscular activity and the increase in intra-abdominal pressure forcing open a previously imperfectly obliterated processus vaginalis. However, it is not clear at this time whether congenital and acquired IIH are basically different from one another, or whether they can be considered to be different manifestations of the same or a similar defect affecting different parts of the inguinal region. Therefore, only the probands with congenital IIH were included in this study.

Previous population studies have suggested that congenital IIH is inherited under a multifactorial threshold model. ${ }^{7-9}$ In order to assess whether the multifactorial threshold model can explain IIH clustering in this data set, two predictions were tested.

First, IIH occurs more commonly in males than in females. This is because of the defect in the abdominal wall occasioned by the descent of the testes into the scrotum in the male and also perhaps because of the protection to the inside of the lower ventral abdominal wall afforded by the uterus in females. Under the multifactorial threshold model, when incidence is different in one sex from the other, risk in relatives depends on the sex of the probands. Contrary to this expectation, the present series shows that the proband's sex was independent of the recurrence risk to the first degree relatives.

Second, the right testis descends later than the left and the processus vaginalis is therefore obliterated later on the right side than on the left side; hence hernia is more frequent on the right than on the left side. If IIH were inherited under a multifactorial threshold model, a higher threshold would be expected for those subjects with left IIH, and consequently a higher risk would be expected in relatives of left IIH than in relatives of right IIH. Similarly, a higher risk would also be expected in the first degree relatives of bilateral probands than in relatives of unilateral probands. This was not the case for either comparison.

Tests of the predictions of the MFT model do not support the assumption of multifactorial inheritance in IIH; rather, we consider autosomal dominant inheritance with incomplete penetrance and sex influence more likely, based on the strong evidence provided by pedigree and segregation analysis.

In families with familial IIH, three important criteria for the model mentioned above are satisfied. First, vertical and male to male transmission of the trait establish the autosomal dominant pattern. Second, some parents of affected subjects are not affected, but they obviously carried and transmitted the deleterious gene. This can be considered as evidence of reduced penetrance. Third, the finding that affected males are much more numerous than affected females suggests that the trait is influenced by sex. This sex influence could be attributed to the anatomical difference between males and females.

Segregation analyses also support autosomal dominant inheritance of IIH. The estimated segregation ratio and penetrance were 0.225 and $0 \cdot 45$, respectively. As expected, the proportion of sporadic cases was unique for $A \times U$ and $U \times U$ (f) matings. In $U \times U$ matings, sporadic cases were estimated to account for most cases.

It is interesting to compare IIH with two other common and extensively investigated congenital malformations, cleft lip with or without cleft palate and neural tube defects, which also result from incomplete closure of the organs involved. Although previous studies have shown that these two conditions have multifactorial inheritance, ${ }^{16-18}$ recent analyses have provided evidence of involvement of major genes. ${ }^{19-25}$ Thus it is plausible to speculate that morphorgenesis may be determined by single genes and complicated by environmental factors. If the IIH gene has a "susceptibility to the environment", it is quite likely that incomplete penetrance or variable phenocopies or both may be present in $\mathrm{IIH}$, and then analyses will result in non-mendelian estimates of the transmission probabilities. This should not necessarily be interpreted as evidence against the major gene hypothesis. ${ }^{26}$

In summary, these data, regardless of the analytical approach, indicate that there is strong statistical evidence that familial IIH is inherited in an autosomal dominant fashion with a reduced penetrance and sex influence.

As mentioned above, IIH occurs more frequently in males than in females, so families with an affected father and son(s) should be expected to be more common than those with an affected mother and son(s). In the group of unaffected carrier parents, however, the carrier fathers are also over-represented although the opposite would be expected. Their inheritance pattern is apparently inconsistent with autosomal dominant inheritance. This observed preponderance of paternal transmission calls for new explanations. We discuss two possibilities here.

First, ascertainment bias might be responsible as affected paternal relatives may be more easily identified since they are more familiar with each other. However, in the other diseases we surveyed by the same methods used for the selection of IIH families, such preponderance was observed in different sexes for different diseases. For example, an excess of maternal transmission was noted in the multiple case families with epilepsy, while an excess of paternal transmission occurred in the multiple case families with cleft lip with or without cleft palate (Gong et al, unpublished data). The fact that male/female ratios of transmitters (normal and affected) shift towards different sexes in different diseases argues against a significant bias of ascertainment in our sample. 
Second, sex specific genomic imprinting could account for the preferential paternal transmission of the IIH gene. We assume that the IIH gene is not expressed or expressed at a low level if it is transmitted from the mother (this kind of imprinting has been referred to as maternal imprinting ${ }^{27}$ ) in contrast to paternal imprinting in which the paternal homologue is inactivated or expressed weakly in the offspring.

Homologous chromosomes may undergo different modifications when they segregate during gametogenesis. These modifications or their effects may persist through embryogenesis and distinguish the maternal and paternal alleles or regions until adult stage. Such imprinted information can apparently result in differential activity of parental alleles. In mammals, the first convincing experimental evidence for genomic imprinting was obtained in marsupial and mouse $\mathrm{X}$ chromosomes. ${ }^{2829} \mathrm{It}$ was found later that this phenomenon was not restricted to the $\mathrm{X}$ chromosomes, as autosomes may also be involved, ${ }^{30}$ and not restricted to the marsupials and mice as in a number of human disorders the phenotypic differences are also related to the parental origin of the disease gene. For example, a congenital and severe form of myotonic dystrophy occurs in 10 to $20 \%$ of myotonic dystrophy families when the gene is transmitted through the mother, ${ }^{31}$ and in 5 to $10 \%$ of families where the Huntington's disease gene is transmitted through the father, a severe, rigid, juvenile form of the disease occurs. ${ }^{32} \mathrm{~A}$ similar effect of genomic imprinting on gene expression has been observed in spinocerebellar ataxia, ${ }^{33}$ seizures, ${ }^{34}$ cerebellar ataxia, ${ }^{35}$ and WiedemannBeckwith syndrome. ${ }^{36}$ The genes involved in these diseases are transmitted in a mendelian manner, but their expression is determined by the sex of the parent transmitting the gene. The pedigrees of these disorders usually show irregular inheritance patterns.

Like the disorders mentioned above, indirect inguinal hernia (IIH) also shows an unusual mode of inheritance. In some families, IIH follows an autosomal dominant pattern, ${ }^{3-5}$ while in most families a monogenic mode of inheritance is not apparent. ${ }^{78}$ Therefore, we are inclined to speculate that a paternal allele is preferentially involved in determining the closure of the inguinal canal. An abnormal paternal gene would result in the failure of closure although the maternal allele there might be normal.

In the mouse there have been numerous cases in which genomic imprinting results in lethality or developmental anomalies, for example, maternal duplication/paternal deficiency for the distal segment of mouse chromosome 2 resulted in flat sided, arch backed, hypokinetic newborn; those mice of the reciprocal type had short, square bodies and broad, flat backs and were notably hyperkinetic $^{37}$; and maternal $\mathrm{X}$ chromosome disomy resulted in developmental failure of the trophoectoderm cell lineage. ${ }^{38}$ In man there have been few reports of congenital malformations whose inheritance is affected by genomic imprinting. One such example is WiedemanBeckwith syndrome (WBS), which is characterised by macroglossia, gigantism, earlobe pits or creases, abdominal wall defects, and an increased risk for the development of tumours. In WBS the paternally transmitted alleles at the WBS locus were supposed to be functionally inactivated to account for the rarity of transmitting males. ${ }^{36}$

In conclusion the fact that most affected males had inherited the IIH gene from their father implicates a role of genomic imprinting in the aetiology of the IIH phenotype. Further follow up of the familial IIH families will allow testing of this hypothesis. Our findings for IIH represent a step towards understanding the developmental basis of this common human genetic disorder.

1 McVay CB. Christopher's textbook of surgery. Philadelphia: Saunders, 1956.

2 Warren RR, Atleson FW. Inheritance of hernia in a family of Holsterin-Fresian cattle. $\mathcal{f}$ Hered 1931;22:345-9.

3 West LS. Two pedigrees showing inherited predisposition to hernia. F Hered 1936;27:449-55.

4 Smith MP, Sparkes RS. Familial inguinal hernia. Surgery 1965;57:807-12.

5 Weimer BR. Congenital inheritance of inguinal hernia. $f$ Hered 1949;40:219-20.

6 Montagu AMF. A case of familial inheritance of oblique inguinal hernia. $\mathcal{F}$ Hered 1942;33:355-6.

7 Sawaguchi S, Matsunaga E, Honna T. A genetic study on indirect inguinal hernia. Ipn f Hum Genet 1975;20:18795

8 Czeizel A, Gardonyi J. A family study of congenital inguinal hernia. Am ₹ Med Genet 1979;4:247-54.

9 Zhang SL. Epidemiological survey of genetic diseases in general population of Sichuan, China. Chengdu: CST

10 Guo YS, Gong YQ, Shao CS, Wei JJ, Chen BX, Jiang Y. A survey for major hereditary diseases in Shandong province. Acta Acad Med Shandong 1931;31:271-5.

11 Falconer DS. The inheritance of liability to certain diseases, estimated from the incidence among relatives. Ann Hum Genet 1965;29:51-76.

12 Carter CO. Genetics of common disorders. Br Med Bull 1969;25:52-7.

13 Reich T, James JW, Morris CA. The use of multiple thresholds in determining the mode of transmission of semi-continuous traits. Ann Hum Genet 1972;36:163-84.

14 Morton NE. Genetic tests under incomplete ascertainment. Am $\mathcal{F}$ Hum Genet 1959;11:1-16.

15 Sun Q, Gong YQ, Guo CH. Family analysis on congenital inguinal hernia. Chin f Med Genet 1991;3:12-13.

16 Carter CO, Evans $\mathrm{K}$. Spina bifida and anencephalus in Greater London. $\mathcal{f}$ Med Genet 1973;10:209-34.

17 Carter CO. Genetics of common single malformations. $\mathrm{Br}$ Med Bull 1976;32:21-6.

18 Lalouel JM, Morton NE, Jackson J. Neural tube malformations: complex segregation analysis and calculation of tions: complex segregation analysis and ca

19 Marazita ML, Spence MA, Melnick M. Genetic analysis of cleft lip with or without cleft palate in Danish kindreds. Am 7 Hum Genet 1984;19:9-18.

20 Marazita ML, Goldstein AM, Smalley SL, Spence MA. Cleft lip with or without cleft palate: reanalysis of a three generation family study from England. Genet Epidemiol 1986;3:335-42.

21 Chung CS, Bixler D, Watanabe T, Kiguchi H, FoghAndersen P. Segregation analysis of cleft lip with or without cleft palate: a comparison of Danish and Japanese data. Am f Hum Genet 1986;39:603-11.

22 Chung CS, Beechert AM, Lew RE. Test of genetic heterogeneity of cleft lip with or without cleft palate as related to race and severity. Genet Epidemiol 1989;6:625-31.

23 Hecht JT, Yang P, Michels VV, Buetow KH. Complex segregation analysis of nonsyndromic cleft lip and palate. Am f Hum Genet 1991;49:674-81.

24 Demenais F, Le Merrer M, Briard ML, Elston RC. Neural tube defects in France: segregation analysis. $\mathrm{Am} \mathcal{F} \mathrm{Med}$ tube defects in France

25 Shaffer LG, Marazita ML, Bodurtha J, Newlin A, Nance WE. Evidence for a major gene in familial anencephaly. WE. Evidence for a major gene in

26 Williams WR, Beutow KH. An explanation of non-Mendelian transmission frequencies in segregation analysis of lian transmission frequencies in segregation analy

27 Hall JG. Genomic imprinting: review and relevance to human diseases. Am $\mathcal{f}$ Hum Genet 1990;46:857-73.

28 Sharman GB. Late DNA replication in the paternally derived $\mathrm{X}$ chromosome of female Kangaroos. Nature 1971;230:231-32

29 Takagi N, Sasaki M. Preferential inactivation of the paternally derived $X$ chromosome in the extraembryonic tissues of the mouse. Nature 1975;256:640-2. 
30 Cattanach BM, Kirk M. Differential activity of maternally and paternally derived chromosome regions in mice. Nature 1985;315:496-8.

31 Harper PS. Congenital myotonic dystrophy in Britain. II Genetic basis. Arch Dis Child 1975;50:514-21

32 Reik W. Genomic imprinting: a possible mechanism for the parental origin effect in Huntington's chorea. $f \mathrm{Med}$ Genet 1988;25:805-8.

33 Zoghbi HY, Pollack MS, Lyons LA, Ferrell RE, Daigner SP, Beaudet AL. Spinocerebellar ataxia: variable age of onset and linkage to HLA in a large kindred. Ann Neuro 1988;23:580-4.

34 Ottman R, Annegers JF, Hauser WA, Kurland LT. Higher risk of seizures in offspring of mothers than of fathers with epilepsy. Am F Hum Genet 1988;43:257-64.

35 Harding AE. Genetic aspects of autosomal dominant late onset cerebellar ataxia. $\mathcal{F}$ Med Genet 1981;18:436-41.

36 Koufos A, Grundy P, Morgan K, et al. Familial Wiedemann-Beckwith syndrome and a second Wilms tumor locus both map to 11 p15.5. Am f Hum Genet 1989;44:711-19.

37 Cattanach BM. Parental origin effects in mice. $f$ Embryol Exp Morphol 1986;97(suppl):137-50.

38 Shao C, Takagi N. A maternally derived X chromosome is deleterious to early mouse development. Development 1990;110:969-75. 